arise from some new form of auroral excitation originating perhaps outside the earth's shadow, or to the recombination of ions which had previously been separated.

The annual period is pronounced, the maximum with us occurring in May or June of each year. This suggests its association with the phenomenon known as the 'Gegenschein,' because it is at this season of the year that the sun is most nearly opposite the place of observation.

On some nights the sky appears to be of great brilliance. The outstanding feature is the smallness of the transmission through the red filter. The auroral green radiation is then relatively bright, but not absolutely large. The main criterion for a brilliant sky is thus the absence of a continuous spectrum.

A memoir embodying the observations made at Mount Stromlo in 1926 and 1927, together with a detailed account of the method of reduction outlined above, is in the printer's hands and will shortly be available for distribution. W. G. Duffintid.

Commonwealth Solar Observatory, Mount Stromlo, Canberra, Australia, Nov. 11.

\section{The Electromagnetic Equations in the Quantum Theory.}

In spite of the great progress made in recent years, the theory of radiation is still in rather an unsatisfactory state. By the methods of Schrödinger it is possible to express the radiation of atoms in the form of electromagnetic waves, but the formulation is quite incomplete, because it fails to give the reaction of the radiation on the emitting system. The theory of Dirac (Proc. Roy. Soc., 114, p. 243) is free from this cardinal fault, but fails to show the relation of radiation to static electric force; it is in fact a valid theory of light, but scarcely an electromagnetic theory. It is of course quite probable that in a complete theory there is no need, or room, for radiation at all, in that the direct interactions of particles according to relativity principles will give all that is required; but radiation must always remain a convenient eliminant, expressive of the effect of a number of particles on a distant one. So it seems not out of place to fit the electromagnetic equations into the general scheme; if they are wrong, it is still interesting to know why Maxwell made the mistake of inventing them !

The following considerations suggest in a natural way how the equations arise. Although by Schrödinger's method it is possible to calculate the radiation scattered in the Compton effect, yet the method is incompetent even to express the idea embodied in the celebrated experiment of Geiger and Bothe (Zeit. für Phys., 32, p. 639), in which it was observed that the directions of scattering of electron and light quantum were absolutely correlated. The simplest way of making it possible to express such an idea is to endow the light with a set of co-ordinates $X, Y, Z, T$, and to have a wave function simultaneously involving both these and the $x, y, z, t$ of the electron. Some such idea is also directly suggested by Dirac's theory, though he makes no use of actual co-ordinates.

The equation determining the behaviour of an electron in a field of radiation is, according to Dirac (Proc. Roy. Soc., 117, p. 610),

$$
\left(p_{0}+a_{1} p_{1}+a_{2} p_{2}+a_{3} p_{3}+a_{4} m c\right) \psi=0 \text {. }
$$

Here the $a$ 's are certain four-rowed matrices, and $p_{1}$ stands for $\frac{h}{2 \pi i} \frac{\partial}{\partial x}+\frac{e}{c} V_{1}$, where $V_{1}$ is the first component of vector potential, while similar meanings connect $p_{2}, p_{3}, p_{0}$ with $y, z, t$. Now $\frac{h}{2 \pi i} \frac{\partial}{\partial x}$ is symbolically the momentum of the electron, and it is therefore natural to regard $e V_{1} / c$ as the momentum of the radiation. The equation then expresses the constancy of momentum in the interaction, and this is just what is used in working out the Compton effect by elementary principles. It is only a step to replace $e V_{1} / c$ by $\frac{h}{2 \pi i} \frac{\partial}{\partial X}$ as the symbolic momentum of the radiation. If now we have a field of radiation far away from the electron, the solution must split into two independent factors, and the radiation by itself will satisfy the equation

$$
\left(-\frac{1}{c} \frac{\partial}{\partial \bar{T}}+\alpha_{1} \frac{\partial}{\partial X}+\alpha_{2} \partial \dot{\partial}+a_{3} \partial \dot{Z}\right) \psi=0 .
$$

When the values of the matrices are substituted, this equation is replaced by four which are exactly Maxwell's equations for free space, combined according to the rules :

$$
\psi_{1}=-i H_{z}, \psi_{2}=H_{y}-i H_{2}, \psi_{3}=E_{z}, \psi_{4}=E_{x}+i E_{v} .
$$

The only difference is that $E$ and $H$ must be real, whereas the $\psi$ 's are usually complex. In a recent paper (Proc. Roy. Soc., 120, p. 621), I pointed out this similarity, but at the time was unable to explain it.

This is, of course, only the germ of the matter, and it leaves many difficulties unsolved. Thus it will be immediately asked how the potentials $V$, which started as coefficients multiplying $\psi$, can be derived from part of the solution for $\psi$ itself. The only answer that can be given is that the same sort of change occurs in other parts of the wave theory, when the reaction on a perturbing system is neglected. It will certainly be necessary to replace the term $m c$ by some function of the co-ordinates, and, among other things, this should lead to an analogue to the classical calculation of electromagnetic mass; but to carry the matter further raises a very fundamental difficulty which $I$ cannot overcome. We have not only two superposed spaces, but also two superposed times, and this is an idea that is very difficult to apprehend, for it, so to speak, dislocates the whole process. This difficulty is not special to the present work, but inevitably occurs in any relativistic representation of more than one particle. Since it may be some time (or should it now be times!) before this trouble is overcome, I have been emboldened to write the present communication, showing the outline of how we may hope that the old waves can be fitted, almost without change, into the new scheme.

The University, Edinburgh,

$$
\text { Jan. } 17 .
$$

\section{The Absorption of X-Rays.}

THE atomic X-ray absorption coefficients of the elements have commonly been represented by simple formulæ of the type $\tau_{a}=k Z^{x} \lambda^{y}$ ( $k$ a factor involving fundamental atomic constants, $Z$ the atomic number of the absorbing element, $\lambda$ the wave-length of the $\mathrm{X}$-rays, $x$ and $y$ exponents not very different from 4 and 3 respectively). These formulæ have been derived in a variety of ways (J. J. Thomson, A. H. Compton, L. de Broglie, H. A. Kramers), and the complete expression for the absorption coefficient of an element over all ranges or X-ray wave-lengths has been represented as the sum of a number of such terms-each term corresponding to the fluorescent excitation of a distinct series or sub-series $\left(K, L_{1}, \ldots\right)$, and dropping out for wave-lengths longer than that of the corresponding absorption edge. Experimental determinations of the absorption coefficients have shown fair agreement with theory, both with respect to the general run of the coefficients on each side of a discontinuity, and to the magnitudes of the discontinuities.

No. 3093, VoL. 123] 